\title{
ADUBAÇÃO DO ALGODOEIRO COM MICRONUTRIENTES E MATÉRIA ORGÂNICA EM SOLOS DE CERRADO(1)
}

FRANCISCO SOLANO DE OLIVEIRA RODRIGUES FILHO (2), Seção de Algodão, e NELSON PAÚLIERI SABINO (2), Seção de Tecnologia de Fibras, Instituto Agronómico.

\section{RESUMO}

Ensaios de campo foram conduzidos no Estado de São Paulo, por dois anos agricolas, 1973/74 e 1974/75, em solos de cerrado, classificados como Latossolo Vermelho-Amarelo fase arenosa, no municipio de Aguaf́, e Podzólico Vermelho-Amarelo variação Laras, no de Ibitiruna, para estudar a aplicação de micronutrientes (zinco, cobre e manganês) e matéria orgânica (esterco de galinha) na cultura do algodoeiro. Não foram verificados efeitos significativos pela aplicação de micronutrientes, embora fornecimentos isolados dos elementos, em associação com uma adubação básica NPK, tenham proporcionado aumentos de 18,30 e $35 \%$ na produção, respectivamente para zinco, cobre e manganês. $O$ esterco de galinha provocou efeito significativo sobre a produção, com aumentos de até $44 \%$, quando utilizado na dose de $800 \mathrm{~kg} / \mathrm{ha}$. Sua aplicação não associada à adubação mineral básica NPK, proporcionou produção de algodão em caroço semelhante ao tratamento sem adủo, no ano agricola 1974/75. Não foi constatado efeito significativo dos micronutrientes e da matéria orgânica, quer sobre as caracteristicas agronômicas, quer sobre as propriedades tecnológicas da fibra.

\section{INTRODUÇĀO}

Embora os solos de cerrado apresentem problemas, condições como a necessidade de terrenos planos e amplos para a introdução da mecanização agricola e a crescente valorização dos terrenos localizados próximo

(1) Da Dissertação para obtenção do título de Meștre em Solos e Nutrição de Plantas, do primeiro autor, pela Escola Superior de Agricultura «Luiz de Queiroz», Piracicaba, em agosto de 1976. Recebido para publicaçāo a 27 de julho de 1982.

(2) Bolsista do CNPq. 
aos centros urbanos consumidores, têm determinado que esses solos, até então marginalizados pela agricultura, passem a ser aproveitados, cada vez com maior freqüência, para a instalação das grandes culturas. $\hat{\mathbb{E}}$, pois, de grande interesse, o estabelecimento de um meio de sua utilização, tornandc-os apropricidos para a agricultura.

Segundo COMISSÃO DE SOLOS (2), JACOMINI (10), BENNEMA (1) e FALESI (5), tais solos têm conteúdo de bases trocáveis, índice de saturação em bases, fósforo assimilável e capacidade de troca de cátions muito baixos; são, ainda, ácidos e de baixa fertilidade, fatores esses, provavelmente, que mais os limitam para a agricultura.

Segundo a literatura nacional, têm sido numerosos os esiorços dos pesquisadores no sentido de estudar os efeitos da adubação na cultura do algodoeiro em diversos tipos de solos. Entretanto, poucos trabalhos trataram do estudo de um meio de utilização dos solos de cerrado: entre eles, citam-se os realizados por McCLUNG et alii (12), FREITAS et alii (7), MIKKELSEN et alii (15), SOUZA et alii (22) e MENDES et alii (13), com resultados bastante discordantes. No exterior, os trabaIhos de adubação do algodoeiro com micronutrientes também são poucos e os resultados encontrados sāo muito variados: NAVARRO (16), YAKOUBOV \& RAKHAMANOV (24), DARGAN \& SAHNI (3), DASTUR \& SINGH (4).

Efeitos da aplicação de matéria orgânica na cultura do algodoeiro são indicados por vários pesquisadores, e a maioria verificou efeito favorável desse material sobre a produção de algodão em caroço, como MENEZES \& ARAÚJO (14), GUIMARĀES (9), SILVA (21), STEPHENS (23), LAL et alii (11) e FERRAZ et alii (6).

O presente trabalho, conduzido durante os anos agricolas de $1973 / 74$ e $1974 / 75$, teve como objetivo verificar a viabilidade de aplicação de micronutrientes (zinco, cobre e manganês) e matéria orgânica (esterco de galinha), associados à adubaçāo mineral NPK, na cultura do algodoeiro, medindo seus efeitos sobre a produção de algodão em caroço, sobre as características agronômicas e sobre as caracteristicas tecnológicas da fibra.

\section{MATERIAL E MÊTODOS}

Os ensaios foram conduzidos durante os anos agricolas de 1973/74 e 1974/75, nas localidades de Aguaí e Ibitiruna em solos classificados, segundo a COMISSÃO DE SOLOS (2), respectivamente como Latossolo Vermelho-Amarelo fase arenosa e Podzólico Vermelho-Amarelo variação) Laras.

Amostras de terra retiradas da camada superficial (0 a $20 \mathrm{~cm})$ e analisadas pela Seção de Fertilidade do Solo do Instituto Agronômico de Campinas, revelaram tratar-se de solos medianamente ácidos a ácidos e de baixa fertilidade, de acordo com os padrões de fertilidade estabelecidos por GARGANTINI et alii (8). 


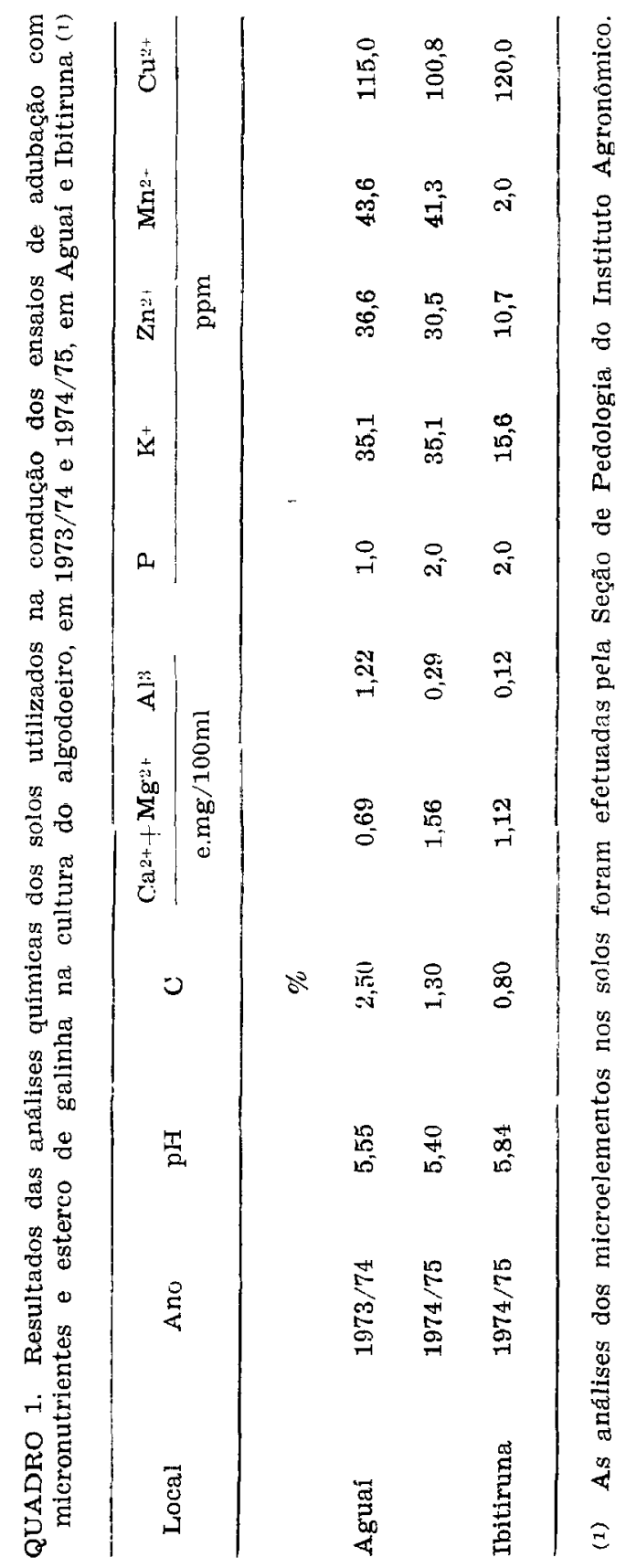

Bragantia; Campinas, 43(2):347-356, 1984 
No ano agrícola de $1973 / 74$, os micronutrientes zinco, cobre e manganês foram aplicados tanto isoladamente, em duas doses, como em mistura entre sí, sempre associados à adubação mineral básica $N_{1} P_{1} K_{1}$. No de $1974 / 75$, os micronutrientes foram aplicados apenas separadamente, embora novamente associados à adubação mineral básica $N_{1} P_{1} K_{1}$.

Os micronutrientes foram fornecidos mediante produtos puros para análise, na forma de sulfatos, nas doses de 5 e $10 \mathrm{~kg} /$ ha de cada elemento, nos anos agrícolas de 1973/74 e 1974/75; neste último ano, para o zinco foi empregada ainda a dose de $15 \mathrm{~kg} / \mathrm{ha}$. Nos tratamentos onde foram aplicadas misturas de micronutrientes, a dose de cada elemento foi $5 \mathrm{~kg} / \mathrm{ha}$. Os micronutrientes foram aplicados junto com o nitrogênio, quando da primeira adubação em cobertura.

Como fonte de matéria orgânica, foi usado o esterco de galinha nas doses de 400 e $800 \mathrm{~kg} /$ ha do material, no ano agrícola de 1973/74, e 400, 800 e $1.200 \mathrm{~kg} / \mathrm{ha}$ em 1974/75. A aplicação do material orgânico f̂oi sempre efetuada juntamente com a adubaçāo mineral básica $N_{1} P_{1} K_{1}$, por ocasião do plantio, procurando-se evitar ao máximo o contato dos adubos com as sementes. No segundo ano agrícola, foi aỏicionado também um tratamento onde se procurou verificar a influência da matéria orgânica isoladamente, empregando-se para tanto a dose de $800 \mathrm{~kg} / \mathrm{ha}$ do esterco de galinha.

O nitrogênio, o fósforo e o potássio foram fornecidos através dos produtos comerciais sulfato de amônio, superfosfato triplo e cloreto de potássio, nas doses de 0,50 e $75 \mathrm{~kg} /$ ha de $\mathrm{N}, 0,80$ e $120 \mathrm{~kg} / \mathrm{ha}$ de $\mathrm{P}_{2} \mathrm{O}_{5}$ e 0,50 e $75 \mathrm{~kg} /$ ha de $\mathrm{K}_{2} \mathrm{O}$, no ano agrícola de $1973 / 74$. No de $1974 / 75$, foi acrescentada mais uma dose de cada elemento, 100 de $\mathrm{N}, 160$ de $\mathrm{P}_{2} \mathrm{O}_{5}$ e 100 de $\mathrm{K}_{2} \mathrm{O}$. As doses básicas de $\mathrm{N}, \mathrm{P}_{2} \mathrm{O}_{5}$ e $\mathrm{K}_{2} \mathrm{O}, 50,80$ e $50 \mathrm{~kg} / \mathrm{ha}$ respectivamente, foram recomendadas com base nas análises químicas dos solos. A mistura de adubos minerais NPK foi incorporada ao solo, no sulco de semeadura, em posição lateral e abaixo do nivel das sementes. A adubação nitrogenada foi parcelada, aplicando-se $10 \mathrm{~kg} /$ ha de $\mathrm{N}$ no plantio e o restante, em duas coberturas, a primeira trinta dias após o plantio e, a segunda, quinze dias depois da primeira.

Foram estudados quinze tratamentos, sendo adotado o delineamento experimental de blocos ao acaso com quatro repetiçōes.

As parcelas experimentais foram constituídas por quatro linhas de $5 \mathrm{~m}$ de comprimento cada uma, sendo consideradas úteis para os estudos apenas as duas linhas centrais de cada parcela. O espaçamento entre linhas foi $0,55 \mathrm{~m}$. Na semeadura, foram utilizadas cerca de trinta-quarenta sementes por metro linear, cobrindo-as com uma leve camada de terra e, no desbaste, foram deixadas quarenta plantas por linha. A variedade empregada foi a IAC-13-1.

Quando mais de $70 \%$ dos capulhos estavam completamente abertos e bem formados, foi realizada a primeira colheita, retirando-se vinte 
amostras de capulhos da parte média das plantas, nas duas linhas centrais. Depois de beneficiadas em máquinas de rolos, essas amostras serviram para estudos das características agronômicas (peso de um capulho, peso de cem sementes e porcentagem de fibra) e das caracteristicas tecnológicas da fibra (comprimento, uniformidade, resistência e complexo finura + maturidade), conforme proposto por SABINO et alii (19).

Decorridos trinta dias da primeira colheita, procedeu-se à segunda, que, somada à primeira e às amostras de vinte capulhos, forneceu a produção total de algodão em caroço por parcela; os resultados, transformados em quilograma/hectare, são apresentados nos quadros 2 e 3.

\section{RESULTADOS E DISCUSSÃo}

No ano agrícola de $1973 / 74$, o ensaio localizado no municipio de Ibitiruna não foi aproveitado devido ao excesso de chuvas na região. Os resultados médios de produção de algodão em caroço cbtidos nos anos agrícolas de 1973/74, no ensaio de Aguaí, e de 1974/75 em ambos os municípios são mostrados nos quadros 2 e 3 respectivamente.

QUADRo 2. Médias dos resultados obtidos no ensaio de adubação do algodoeiro com micronutrientes e esterco de galinha, realizado em sclo de cerrado de Aguaí (SP), em 1973/74

Produçãc

Tratamentos

Algodão em caroço

Relativa .

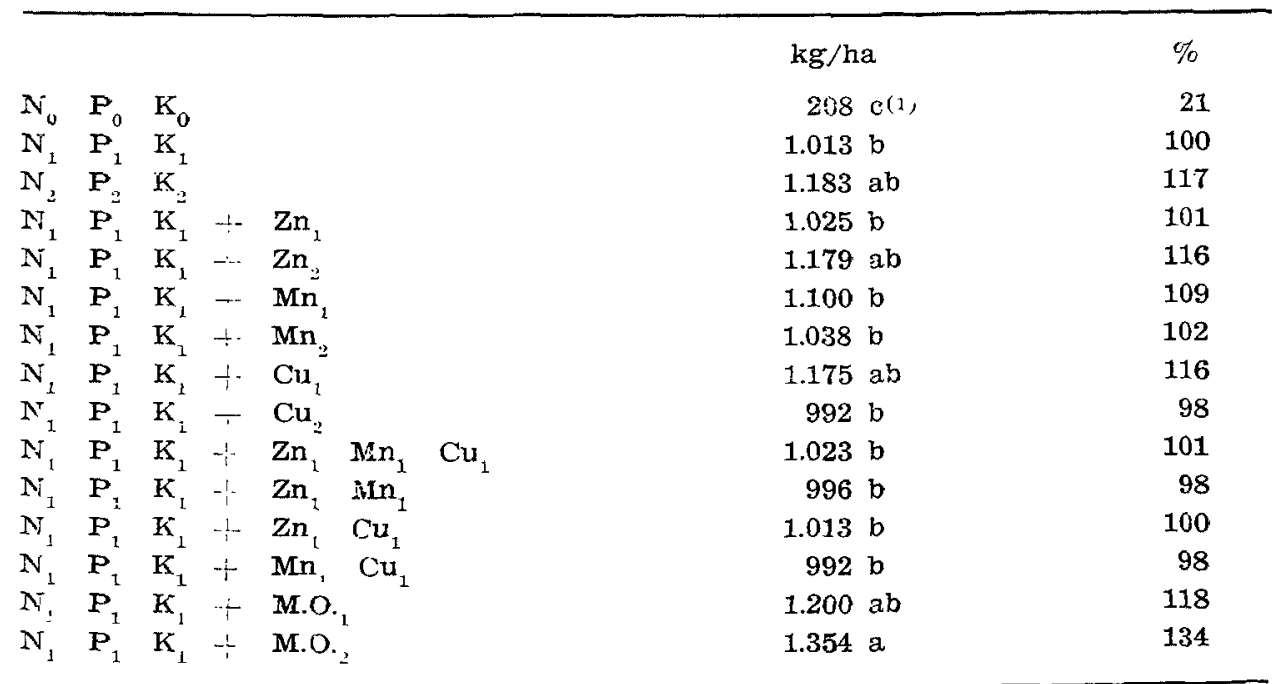

(1) Médias seguidas de letras iguais não diferem estatisticamente entre si (Duncan, $5 \%$ ). 
QUADRO 3. Médias dos resultados obtidos nos ensaios de adubação do algodoeiro com micronutrientes e esterco de galinha, realizados em solos de cerrado em Aguaí e Ibitiruna (SP), em 1974/75

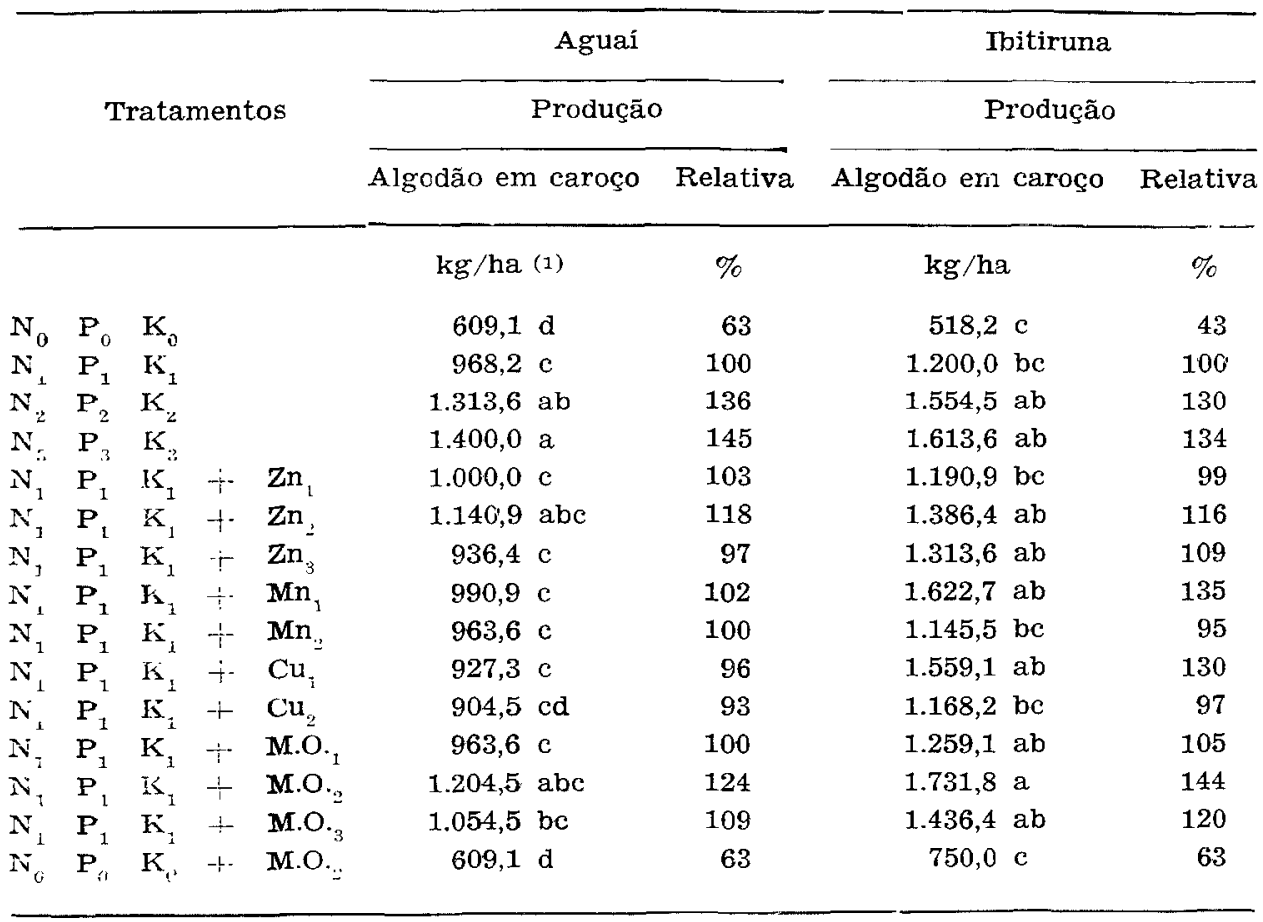

(1) Médias seguidas de letras iguais não diferem estatisticamente entre si (Duncan, $5 \%$ ).

A avaliação dos efeitos dos micronutrientes, aplicados de forma individual ou em misturas entre si, e da matéria crgânica associada ou não à adubação mineral básica, foi feita em relação à adubação mineral básica $\mathrm{N}_{1} \mathrm{P}_{1} \mathrm{~K}_{1}$.

Não houve efeito estatisticamente significativo devido à aplicaçâo de micronutrientes em Aguaí, apesar de a aplicação de zinco na dose

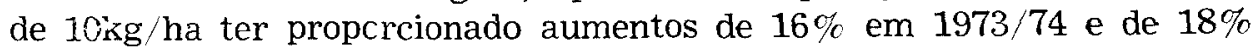
em 1974/75. Os efeitos do cobre e manganês não foram consistentes de ano para ano.

Tanto em Ibitiruna como em Aguaí, no ano agrícola de 1974/75, o zinco provocou aumento na produção com a dose de $10 \mathrm{~kg} / \mathrm{ha}$ e um decréscimo com a de $15 \mathrm{~kg} / \mathrm{ha}$, o que parece indicar que a dose mais elevada do zinco pode ter sido excessiva, em ambas as localidades.

O manganês e o cobre, embora não apresentando efeitos significativos, provocaram um acréscimo de produção, em Ibitiruna, de 35 e 
$30 \%$, respectivamente, com o emprego da dose de $5 \mathrm{~kg} / \mathrm{ha}$ de carda elemento.

Os resultados do presente trabalho são diferentes dos obtidos por DARGAN \& SAHNI (3), YAKOUBOV \& RAKHAMANOV (24), porém confirmam os alcançados por McCLUNG et alii (12), MENDES et alii (13), SILVA \& FALIERI (20), FREITAS et alii (y), os quais não verificaram efeito significativo da aplicação de micronutrientes na cultura do algodoeiro, quer aplicando-os isoladamente, quer em mistura, associados à adubação mineral NPK. No presente estudo, a aplicação de mistura de micronutrientes, em 1973/74, chegou inclusive a deprimir a produção em alguns casos, contrariando os resultados obtidos por NAVARRO (16), que verificou efeito significativo da aplicação de micronutrientes.

As aplicações das doses de 400 e $800 \mathrm{~kg} /$ ha de esterco de galinha, no ano agrícola de 1973/74, em Aguaí, proporcionaram aumentos de produção de 18 e $34 \%$ respectivamente. A dose maior do esterco diferiu estatisticamente da adubação mineral básica, não tendo havido, entretanto, diferença entre as duas doses empregadas. Fato semelhante ocorreu em 1974/75, sendo, porém, menores os aumentos proporcionados por esse material orgânico.

Em Ibitiruna, no ano agricola de 1974/75, o esterco de galinha também proporcionou efeito significativo sobre a produção de algodâo em caroço, obtendo-se o melhor resultado com a dose de $800 \mathrm{~kg} / \mathrm{ha}$.

Tanto em Aguai como em Ibitiruna, a dose de $1.200 \mathrm{~kg} /$ ha de esterco de galinha deprimiu a produção em relação à de $800 \mathrm{~kg} / \mathrm{ha}$.

Os resultados aqui relatados vêm confirmar o efeito benéfico da aplicação da matéria orgânica ao solo, sobre a produçāo do algodoeiro, obtidos por MENEZES \& ARAÚJO (14), STEPHENS (23), LAL et alii (11), GUIMARÃES (9) e SILVA (21).

Outro fato que merece destaque neste estudo, ocorrido nas duas localidades, no ano agrícola de 1974/75, diz respeito à produção do algodoeiro quando foi aplicada a matéria orgânica isolada, rão associada à adubação mineral básica $\mathrm{N}_{1} \mathrm{P}_{1} \mathrm{~K}_{1}$ : a produção de algodão tem caroço obtido com esse tratamento foi semelhante à obtida com o tiatamento sem adubo (testemunha absoluta), indicando que, para que seja alcançado sucesso na aplicação da matéria orgânica, esta deve ser aplicada ao solo juntamente com a adubaçāo mineral NPK.

Pelos resultados de produção mostrados nos quadros 2 e 3 , pode-se observar que todos os tratamentos, com exceção daquele onde se aplicou matéria orgânica somente, diferiram da testemunha sem adubo ou testemunha absoluta.

Por outro lado, os niveis de produtividade alcançados com a aplicação de fertilizantes NPK em solos do tipo dos utilizados neste estudo, tornam a cultura anti-econômica, se não for realizada a calagem, mesmo 
que o $\mathrm{pH}$ esteja em uma faixa considerada adequada para a cultura, pois os teores de cálcio + magnésio, detectados pelas análises dos solos, são relativamente baixos.

No tocante às características agronômicas e às características tecnológicas da fibra do algodoeiro, tanto os micronutrientes como a matéria orgânica não proporcionaram efeitos significativos, confirmando os resultados obtidos por DASTUR \& SINGH (4), DARGAN \& SAHNI (3) e NAYAK $(\mathbf{1 7}, \mathbf{1 8})$.

\section{CONCLUSÕES}

Os resultados obtidos permitiram as seguintes conclusōes:

a) A aplicação dos micronutrientes zinco, cobre e manganês, de forma individual ou em mistura, associados à adubação mineral NPK, não proporcionou efeito significativo sobre a produção do algodoeiro, embora aplicações individuais de zinco tenham aumentado a produção até $18 \%$ e de cobre e manganês 30 e $35 \%$ respectivamente.

b) A matéria orgânica, sob a forma de esterco de galinha e empregada junto com adubação mineral NPK, provocou efeitos significativos sobre a produção do algodoeiro, com aumentos de produção de até $44 \%$, quando utilizada na dose de $800 \mathrm{~kg} / \mathrm{ha}$. O aumento ca dose para $1.200 \mathrm{~kg} /$ ha não trouxe benefícios à produção.

c) A aplicação de esterco de galinha isolado proporcionou produções de algodão em caroço semelhantes às obtidas com o tratamento sem adubo, indicando que esse material orgânico não foi suficiente quando aplicado na ausência de adubação mineral NPK.

d) Os micronutrientes usados e o esterco de galinha não afetaram as características agronômicas (peso de um capulho, peso de cem sementes e porcentagem de fibra) e as caracteristicas tecnológicas da fibra (comprimento, uniformidade, resistencia e complexo finura + maturidade).

e) Os baixos níveis de produção obtidos podem ser uma indicativa da necessidade de cutras práticas nesse tipo de solo.

\section{SUMMARY}

\section{EFFECTS OF NUTRIENTS AND ORGANIC MATTER ON COTTON PRODUCTION}

The effects of micronutrients $(\mathrm{Zn}, \mathrm{Cu}$ and $\mathrm{Mn})$ and organic matter (chicken-manure) associated with the basic NPK fertilization, on cotton production, yield properties and fiber properties were studied in field tests conducted during the years of 1973 to 1974 , in Ibitiruna and Aguaí in the State of São Paulo, Brazil. The soils utilized presented medium acidity and low fertility, according to chemical 
analysis, and the cotton variety tested was IAC 13-1. Complete randomized block designs were used, with four replications. The following conclusions were from the results: a) The application of micronutrients did not show a statistically significant effect on cotton yield, although an increase, up to $35 \%$, on cotton yield was observed. b) The effect of the application of organic matter on cotton yield showed a significant effect, but dependent on the year, with increases in cotton yield from 5 to $44 \%$. c) No significant effects of micronutrients or organic matter were detected on yield properties and fiber technology properties. d) The application of the organic matter alone, without association with the NPK fertilizers, showed the same production of the treatment without any fertilization. e) The low levels of productivity obtained might be an indication of lack of other management practices,

\section{AGRADECIMENTOS}

Os autores agradecem aos senhores Natálio Sabino e Guilherme Rehder a colaboração prestada na execução dos experimentos em suas propriedades, e à Seção de Pedologia, as análises dos microelementos nos solos.

\section{REFERENCIAS BIBLIOGRAFICAS}

1. BENNEMA, F. Características químicas e físicas de latossolos sob vegetação de cerrado. In: BRASIL. Ministério da Agricultura. Serviço de Informação Agricola. Recuperação do Cerrado. Rio de Janeiro, 1964. p.137-143. (Estudos brasileiros, 21)

2. BRASIL. Ministério da Agricultura. Levantamento de reconhecimento dos solos do Estado de São Paulo: Contribuição à Carta de Soios do Brasil ... Rio de Janeiro, Centro Nacional de Ensino e Pesquisas Agronômicas, 1960. 634p. (Boletim do Serviço Nacional de Pesquisas Agronômicas, 12)

3. DARGAN, K.S. \& SAHNI, V.M. Studies on the effect of micronutrients on the yield and economic characters of American cotton. Indian Cotton Journal, $19: 373-375,1965$.

4. DASTUR, R.H. \& SINGH, K. A study of the effect of trace elements on the growth and yield of American cottons. Indian Coiton Growing Review, 7:57-66, 1953.

5. FALESI, I.C. Levantamento de reconhecimento detalhado dos solos da Estrada de Ferro do Amapá. Belém, Instituto de Pesquisas e Experimentação Agropecuária do Norte, 1964. 53p.

6. FERRAZ, C.A.M.; FUZATTO, M.G.; GRIDI-PAPP, I.L. Influência da adubação com torta de café na germinação do algodoeiro. Bragantia, Campinas, 22:131-138, 1963.

7. FREITAS, L.M.M.; McCLUNG, A.C.; LOTT, W.L. Experimento de adubação em dois solos de campo cerrado. New York, IBEC Research Institute, 1960. 32p. (Boletim, 21)

8. GARGANTINI, H.; COELHO, F.A.S.; VERLENGIA, F.; SOARES, E. Levantamento de fertilidade dos solos do Estado de São Paulo. Campinas, Instituto Agronômico, 1970 . 31p.

9. GUIMARÃES, A.A. Experimentação agrícola em Minas Gerais. Boletim de Agricultura, Belo Horizonte, 8(11/12):198, 1959.

10. JACOMINI, P.K.T. Consideraçōes gerais sobre alguns solos de cerrado. In: BRASIL. Ministério da Agricultura. Serviço de Informação Agrícola. Re- 
cuperação do Cerrado. Rio de Janeiro, 1964. p.131-136. (Estudos brasileiros, 21)

11. LAL, P.; GARG, O.P.; BAJPAI, M.R. Effect of source and mode of nitrogen application on cotton (Gessypium hirsutum L.). Residual effect on the following crop (oats) and soil organic matter. Soil and Fertilizers, 37:140, 1974.

12. MCCLUNG, A.C.; FRETTAS, L.M.M.; MIKKELSEN, D.S.; LOT, W.L. Cotton fertilization in campo cerrado soils of the State of São Paulo, Brazil. New York, IBEC Research Institute, 1961. 31p.

13. MENDES, H.C.; GALLO, J.R.; ABRAMIDES, E. Nutrição do algodoeiro. III - Efeitos de micronutrientes em plantas vegetando am vasos. Bragantia, Campinas, 19:LXXVII-LXXXIV, 1960. (Nota, 17)

14. MENEZES, W.C. \& ARAŨJO, W.A. Ensaios de adubação do algodoeiro na Estação Experimental de Sete Lagoas. In: BRASIL. Ministério da Agricultura. Serviço de Informação Agricola. Recuperação do Cerrado. Rio de Janeiro, 1964. p.25-44. (Estudos brasileiros, 21)

15. MIKKELSEN, D.S.; FRETTAS, L.M.M.; MCCLUNG, A.C. Efeitos da calagem e adubação na produção de algodão, milho e soja em tress solos de campo cerrado. São Paulo, Instituto de Pesquisas IRI, 1963. 48p. (Boletim, 29)

16 NAVARRO, R.P. Respuesta del algodonero a la applicación de elementos menores al suelo. Acta Agronomica, Colombia, 11(3/4):131-145, 1961.

17. NAYAK, H.R. Effect of manuring on quality of cotton. Indian Cotton Growing Review, 15:303-307, 1961.

18. Response of fiber properties and agronomic character of cotton to different doses of farm yard manure. Indian Cotton Growing Review, 7:292-296, 1953.

19. SABINO, N.P.; LAZZARINI, J.F.; GRIDI-PAPP, I.L.; FUZZATO, M.G.; GROSSI, J.M.M. Estudo de amostragem de capulhos em canteiros experimentais de algodão. Bragantia, Campinas, 34:163-169, 1975.

20. SILVA, A.F. \& FALIERT, J. Relatório do Setor de Algodão da Estação Experimental de Sete Lagoas do Instituto de Pesquisa Agropecuária Centro-Oeste. 1969. (Mimeografado)

21. SILVA, N.M. Estudo preliminar de emprego de torta de mamona associada à adubação mineral do algodoeiro. Campinas, Instituto Agronômico, 1971. 8p. (Projeto BNDE/ANDA/CIA, 10)

22. SOUZA, L.S.; SOBRAL, L.F.; ALVES, E.J. Efeitos de N, F, K, S e micronutrientes na produção do algodoeiro. In: INSTITUTO DE PESQUISAS AGROPECUARIAS DO LESTE, Cruz das Almas. Solos. Cruz das Almas, IPEAL, 1973. p.35-47. (Boletim técnico, 20)

23. STEPHENS, D. Effects of different nitrogen treatments and potash, lime and trace elements on cotton on Buganda Clay Loam Soil. Cotton Growing Review, 44:313, 1967.

24. YAKOUBOV, A.M. \& RAKHAMANOV, R.R. Effect of microelements on yield and chemical constitution of cotton. Cotton Growing Review, 47:191-197, 1970. 